\title{
Carnets
}

Revue électronique d'études françaises de l'APEF

Deuxième série - 19 | 2020

Petite fabrique d'interprètes

\section{Il Était une fois un arbre...}

À propos de A Árvore das Palavras [L'Arbre à Paroles] de Teolinda Gersão

\section{Maria de Fátima Marinho}

\section{CpenEdition}

Journals

Édition électronique

URL : http://journals.openedition.org/carnets/11251

DOI : 10.4000/carnets. 11251

ISSN : 1646-7698

Éditeur

APEF

Référence électronique

Maria de Fátima Marinho, « II Était une fois un arbre... », Carnets [En ligne], Deuxième série - 19| 2020, mis en ligne le 31 mai 2020, consulté le 23 décembre 2020. URL : http://journals.openedition.org/ carnets/11251; DOI : https://doi.org/10.4000/carnets.11251

Ce document a été généré automatiquement le 23 décembre 2020.

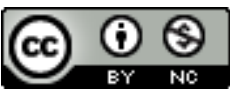

Carnets est mis à disposition selon les termes de la licence Creative Commons - Atribution - Pas d'utilisation commerciale 4.0 International. 


\title{
Il Était une fois un arbre...
}

\author{
À propos de A Árvore das Palavras [L'Arbre à Paroles] de Teolinda Gersão
}

\author{
Maria de Fátima Marinho
}

1 Depuis des temps immémoriaux, l'arbre a toujours joué un rôle que l'on peut difficilement ignorer. À l'époque où la nature imprimait son rythme à la vie de tous les jours et où l'homme se distançait à peine du rythme global de l'univers environnant, les sens attribués à l'arbre recouvraient un ensemble extrêmement varié de stéréotypes dont chacun était lié à une fonction cruciale dans le cycle de la vie. Axe du monde, symbole de la création dans plusieurs religions et cultures, l'arbre, avec ses racines dans la terre et ses branches dans le ciel, ressemble à l'homme et à sa nature ambiguë, à la fois céleste et tellurique, angélique et pécheresse. Le rôle de l'arbre dans l'interprétation des phénomènes compliqués tels que celui de la mort et de la vie, voire de la résurrection, dans le christianisme, ainsi que la difficulté à le cataloguer toujours de la même façon favorisent l'apparition, dans la littérature, de textes légitimant les diverses interprétations, en jouant sur les croyances, souvent à l'état subliminal, qui conjuguent la religiosité avec la pratique culturelle et la vie quotidienne du sujet.

2 L'idée de continuité et de successivité que semble indiquer la renaissance printanière, conjuguée avec la représentation de la lignée familiale dans les arbres généalogiques, favorise des lectures qui ne peuvent que réitérer l'idée selon laquelle la racine et ses ramifications ressemblent dangereusement à la vie humaine et à ses desseins les plus secrets.

3 Partant de cette certitude, tantôt simple, tantôt complexe, voire embarrassante, les traditions culturelles de différents peuples et de différentes régions ont utilisé le symbole de l'arbre comme moteur d'intrigues se déroulant dans le sillage d'une multitude de relations interpersonnelles.

4 L'ambivalence, présente dans de nombreux textes littéraires, constitue un des points centraux du roman que nous nous proposons d'étudier. A Árvore das Palavras, de Teolinda Gersão, publié en 1997, place dans la bouche d'une petite fille, qui devient adolescente puis jeune adulte, le récit d'une expérience au Mozambique, ancienne colonie portugaise. Fille de deux colons blancs, elle se sent assimilée à l'Afrique et à sa nourrice noire, sentiment qu'elle partage avec son père, Laureano, mais que sa mère 
rejette catégoriquement. Celle-ci s'est mariée grâce à une petite annonce et elle arrive en Afrique la tête pleine de rêves de richesse, rêves qu'elle ne parviendra pas à réaliser dans sa vie de modeste couturière. L'inadaptation d'Amélia, la mère, crée un fossé entre sa fille, Gita, et elle, et la pousse à chercher le bonheur en Australie (lieu distant, utopique), entreprise dont on ignore le dénouement. Le voyage (véritable fuite) en Australie, pour suivre un homme qu'elle a également rencontré par une petite annonce, est une répétition de la première histoire et laisse entrevoir de nouvelles déceptions. Sur fond de frustration et de perte, d'une part, et de maturation en relation étroite avec la nature et le milieu africain, d'autre part, l'arbre, d'ailleurs présent dès le titre, revêt plusieurs sens qui vont de la simple évocation à l'interférence à des moments-clé de l'intrigue et de la consolidation des personnages.

5 La présence de l'arbre dans le paysage, faisant partie de la vie de tous les jours et s'immisçant dans l'expérience de vie des sujets, révèle une ambivalence fondamentale qui se traduit de nombreuses façons dans le texte de livres essentiels pour la construction de l'imaginaire de l'individu occidental, tels que la Bible ou le Coran. Hésitant entre l'arbre de la vie et l'arbre du péché originel, l'homme (et la femme) répète(nt) inconsciemment le paradigme originel, que l'écrivaine portugaise Teolinda Gersão a si bien su exploiter.

6 L'interdit implicite dans les conseils de Dieu, que l'on trouve dans les textes sacrés, engendre la notion de faute et instaure la séduction de la rupture et la transgressions de notions, apparemment si simples, telles que le temps et ses corrélats (passé nostalgique ou nié et futur à peine entrevu) :

Tu peux manger de tous les arbres du jardin. Mais de l'arbre de la connaissance du bien et du mal tu ne mangeras pas, car, le jour où tu en mangeras, tu mourras certainement. (Gen.2, 16-17: 10) / Nous dîmes : « Adam, séjourne dans le paradis, en compagnie de ton épouse. Mangez [de ses fruits] en toute quiétude, partout où vous le désirez. Mais n'approchez pas de cet arbre : vous seriez du nombre des injustes » (Le Coran, Sourate II, $35: 52$ ).

7 La désobéissance à l'ordre donné par la divinité implique le châtiment et la malédiction éternelle et donne lieu à la construction de l'homme que nous connaissons, fruit de la voix tentatrice du diable :

Vous ne mourrez pas! Mais Dieu sait que, le jour où vous en mangerez, vos yeux s'ouvriront et vous serez comme des dieux, qui connaissent le bien et le mal. (Gen.3. $4-5: 11$ ) / Votre Seigneur ne vous a interdit cet arbre que pour que vous ne soyez ni anges, ni immortels. (Le Coran, Sourate VII, $20: 468$ )

8 Ainsi pouvons-nous comprendre la contradiction insurmontable qui prévoit l'existence d'arbres maudits tels que l'arbre zaqqum (arbre de l'enfer) qui, selon Le Coran, « sera la nourriture du pécheur " (Sourate XLIV, $44: 1580$ ), et d'un arbre à feuilles persistantes auquel « tout ce qu'il fait réussi » (Ps.1-3: 654).

9 L'apologue de Yotam, raconté dans le Livre des Juges (9, 8-15:258), de même que le rêve de Nabuchodonosor et son interprétation (Daniel 4: 1195-1197), semblent appropriés pour bien comprendre le rôle de l'arbre dans le roman en question. Le défi de la détention du pouvoir lancé aux arbres (Livre des Juges) et l'acceptation du buisson d'épines ne manquent pas d'être symptomatiques : le refus de l'olivier, du figuier et de la vigne, compte tenu des dommages résultant de la perte respective de l'huile d'olive, des figues et du vin, et l'acceptation du buisson d'épines (qui ne possède rien d'autre) peuvent vouloir indiquer les contingences de l'exercice du pouvoir et la transposition dans des univers secondaires qui se distinguent à peine dans le jeu de miroirs du texte. 
De même, l'arbre bénéfique, source d'abondance dans le rêve de Nabuchodonosor, que la sentence du Vigilant semble vouloir détruire, signifie, selon Daniel, la prévalence du pouvoir de Dieu sur toute chose, ce qui implique, au niveau textuel, l'impondérabilité de la détention d'une des forces qu'ils se disputent éternellement : Amélia et Laureano sous les yeux de la petite Gita, témoin obligatoire et actant imprévu. La conclusion du personnage biblique Daniel démontre l'impuissance de l'homme, même s'il s'agit d'une impuissance contrôlée :

Sept temps passeront sur toi, jusqu'à ce que tu aies appris que le Très Haut a domaine sur le royaume des hommes et qu'il le donne à qui lui plaît (Daniel 4, 22 :

1196)

La transmutation symbolique des sujets en arbres, annoncée par Gita, et l'obtention du pouvoir qui en découle, peut se lire comme l'image réfléchie et à peine déformée des sens ancestraux de l'arbre et de sa corrélation avec les sujets :

Ficava-se muito tempo debaixo da árvore, encostado ao tronco, e como eu disse, a gente transformava-se em árvore. Ou também em pássaro, embora voar fosse mais difícil. Mas ser as coisas era fácil. Porque de repente se tinha na mão a raiz de tudo o que era vivo. (Gersão, $1997: 21)^{1}$

11 Le « jardin sauvage » (Gersão, $1997: 11)$ dont parle Gita dès le début marque la distance irréductible entre Laureano et Amélia (le père et la mère, que, bizarrement, Gita désigne toujours par leur prénom sans jamais utiliser le lien de parenté qui les unit). Tandis que le premier « plantou ele mesmo um rícino, ao fundo do quintal » (ibid. : 13) ${ }^{2}$, Amélia déteste ce jardin qui la rapproche des Noirs plutôt que des Blancs. Le discours de Gita semble jaillir naturellement d'une matrice primordiale, comme si "parler » était un besoin vital de survie face à un monde hostile. L'arbre à paroles du titre réapparaît presque à la fin du roman, où il signifie la dimension de l'ensemble d'une vie :

Ela crescia nos sonhos, digo a Roberto enquanto pintamos o cartaz. A árvore das palavras. Para contornar o seu tronco seriam precisas nove luas. E cada folha era extensa como um voo de pássaro. (Gersão, $1997: 218)^{3}$

12 Cet arbre, qui, ici, s'épanouit, a plusieurs avatars au cours du texte. Ceux-ci s'immiscent subrepticement dans le discours et présentent des caractéristiques qui le rapprochent beaucoup de l'homme et de sa double nature : sacrée et démoniaque.

13 Si nous analysons le rôle de l'arbre dans le texte qui nous occupe, nous remarquons les divers indices qui sont transmis progressivement et qui culminent dans l'importance du discours en tant qu'élément structurant la personnalité et son intégration dans un milieu donné.

14 Commençons par les diverses références à l'ombre des arbres, dont la fonction première est de tenter d'établir une réalité mouvante, sans consistance, correspondant aux sentiments discordants des personnages :

(...) a silhueta esgalgada das árvores, a Terra é um planeta desolado e morto, boiando. (ibid. : 34) ; seguiria até ao fim da cidade de cimento e continuaria a andar, no meio da sombra rala, das árvores dispersas (ibid.: 47) ; Pisando, no chão, as sombras esguias, movediças, dos coqueiros. (ibid. : 147) ${ }^{4}$.

L'instabilité de l'ombre, analogue à celle du sujet, légitime la recherche de refuges, simulacres des points d'appui d'un sujet en pure perte, comme le démontre Carlo Ossala dans une étude publiée en 2011. S'asseoir sous l'arbre (position récurrente dans le roman) reproduit des gestes semblables à ceux décrits dans des textes de la doctrine 
bouddhiste (Crépon, 1991 : 182-183), hindoue, chrétienne ou musulmane et renforce la capacité de réflexion et l'interdépendance entre les deux mondes en confrontation :

Ou sentava-me debaixo da árvore do quintal e falava com o vento e as folhas. A

árvore abanava os ramos e eu pensava : a árvore das palavras. (Gersão, $1997: 39)^{5}$.

La présence constante de l'arbre dans la vie de tous les jours, lieu de méditation ou refuge (« escondi-me atrás da árvore mais próxima », ibid.: $235^{6}$ ), ne signifie pas qu'il s'agisse toujours du même arbre, comme nous l'avons d'ailleurs déjà souligné au début de ce petit essai. Dans le roman de Teolinda Gersão apparaissent des acacias, des jacarandas, des anacardiers, des manguiers, des cocotiers, des papayers, des arbres à caoutchouc, ce qui n'a rien d'étonnant dans un décor africain. Il faut surtout tenir compte du rôle de l'acacia étant donné qu'il symbolise la victoire sur la mort (Encyclopédie, 1996:5) et que cette victoire est fondamentale au niveau de la structure profonde du discours, du jeu avec l'explicite de l'action et l'implicite des sens cachés qui déterminent les comportements et les sentiments des personnages.

Pourtant, avant d'atteindre le stade de dépassement, qu'indique le départ de Gita pour Lisbonne à la fin du roman, nous pouvons analyser quelques-uns des détails semés au fil du roman qui contribuent à la consolidation du sens en attribuant divers sens à l'arbre.

Si l'inclusion d'un vers de l'hymne de la Mocidade Portuguesa (organisation semiguerrière de jeunes sous l'État Nouveau de Salazar) renvoyant à la vie dans la Métropole (le Portugal continental) vise à montrer, par la caricature et en recourant à la synecdoque, la rigueur de l'éducation et la répétition acritique du discours normalisateur et asphyxiant ("Tronco em flor estende os ramos à mocidade que passa ", Gersão, 1997 : 2047), l'affirmation ostentatoire de l'évidence devient le noyau agglutinant de la relation intrinsèque avec le corps, relation exprimée au moyen d'affirmations apparemment simples et tautologiques: "Tu és bom como as árvores são árvores e a chuva é a chuva. » (ibid. : 29) ${ }^{8}$ ou

Eu sou, dizia a árvore agitando os ramos, a semente abrindo no escuro, a água

apodrecendo nas lânguas, a floresta dormindo. Eu sou. (ibid. : 63)9.

19 La fusion intime avec le corps, "O corpo era a árvore e o corpo era o vento " (ibid. : 16) ${ }^{10}$, légitime l'inclusion de la danse comme moyen de médiatiser des sentiments aussi disparates que la rébellion ou la peur et de réévaluer la relation avec le passé et le désir d'influencer une utopie à venir.

20 Selon Gita, «A dança é isso, um modo mais intenso de existir. As árvores dançam, as folhas dançam, a chuva dança, os animais dançam, o sol e a lua dançam. » (Gersão, $1997: 29-30)^{11}$. C'est la seule explication possible au fait que la protagoniste refuse de faire de la danse classique, danse artificielle, construite, trop rigide, inadaptée aux rythmes naturels et en opposition avec la synchronie sous-entendue dans cette affirmation : « eu continuo a dançar descalça, batendo as palmas com as mãos, debaixo das árvores do quintal » (ibid. : 73) ${ }^{12}$.

21 La liberté que la danse africaine présuppose, contrairement à la normalisation absolue qu'implique la danse classique, sous-entend le besoin de transgression déjà prévue dans l'épisode biblique / coranique où Ève viole le tabou et provoque la désobéissance d'Adam. La certitude que l'on ne peut contrôler la naissance et la croissance des arbres, exprimée dans des phrases telles que : « não se podem domesticar as casuarinas, nem os coqueiros, nem os jacarandás " (ibid.: 55$)^{13}$, révèle l'intime corrélation entre les hommes et la nature (les arbres), imprimant à celle-ci (ceux-ci) des caractéristiques qui sont normalement l'apanage de ceux-là. 

jouent un rôle crucial difficile à ignorer, sont chose courante. Dans l'Évangile selon Saint Marc, un aveugle ayant récupéré la vue s'exclame: "J'aperçois les gens, c'est comme si c'était des arbres à marcher» (Marc 8, $24: 1340)$. Et les jugements de valeur où il est question d'arbres pour parler des hommes ne sont pas rares: "Ainsi, tout arbre bon donne de bons fruits, tandis que l'arbre mauvais donne de mauvais fruits.» (Mat. 7, $17: 1298$ ). personnages et transposer des sentiments, des aspirations et des craintes de l'un à l'autre :

Levanto a cabeça para as acácias da rua por onde agora caminhamos, como se elas nos pudessem dar uma resposta, como se pudessem indicar-nos um lugar mais resguardado do que atrás de uma cortina de folhagem. (Gersão, $1997: 213$ );

0 vento sopra lá fora, as árvores arrepiam-se de medo (ibid. : 99);

Mas se chorasse era pior, sentiu tirando da mala um lenço de papel, era como se o mundo risse dela, os guarda-sóis, as casas, os barcos, as árvores, as pessoas, sobretudo as pessoas rissem dela. (Ibid. : 107) ${ }^{14}$.

Le rire, objet de grande méfiance au Moyen-Âge (Tomasset, 2011 : 258-259), provoque ici un sentiment de gêne révélant une âme confuse et déséquilibrée (ibid. : 259), à qui seule la parole permet de retrouver l'équilibre.

Cette transposition, idéale pour faire passer l'information, suggérant plutôt qu'elle n'affirme, se révèle dans les détails les plus infimes tels que celui de l'oiseau qui tombe de l'arbre (" Um pássaro que cai da árvore porque não levantou voo a tempo. », Gersão, $\left.1997: 229^{15}\right)$ et qui transporte le lecteur sur le terrain mouvant de l'incapacité du sujet à construire son propre destin.

26 L'importance de l'arbre des ancêtres (ibid.: 28), conjuguée avec celle de l'arbre à paroles, dont il a déjà été question et qui légitime les paroles de Gita et des autres personnages (même s'il s'agit pour ceux-ci d'un discours rapporté), nous incite à faire des lectures sacrées et telluriques. Le silence apparent de Gita, dont le discours ressemble à une sorte de monologue intérieur évocateur, finit par détenir un pouvoir qui provient de la régulation et du contrôle de la parole (Tomasset, 2011:262) et qui apparaît comme fondamental pour interpréter le sens inhérent à un sujet qui se vide dans la dissolution de son univers primordial (celui de l'enfance) :

Às vezes essa árvore [a das palavras] reaparecia nos sonhos : crescia à beira de um rio e tinha ramos que chegavam ao céu. (Gersão, 1997 : 39), tal como « o corpo [que] ligava a terra ao céu. » (Gersão, $1997: 17)^{16}$.

La fusion de l'arbre et du corps, l'insistance sur ces éléments comme maillons de liaison entre le céleste et le sous-terrain, montrent l'importance que revêt la sagesse dans les rapports entre le sujet et son environnement, y compris les autres sujets qui entrent obligatoirement en contact avec lui. Le lien entre l'arbre et la sagesse est déjà manifeste dans le Livre des Proverbes (« C'est un arbre de vie pour qui la [sagesse] saisit, celui qui la tient devient heureux », Prov. 3, 18: 804) et on le devine dans la description de la Jérusalem messianique à la fin de l'Apocalypse :

Au milieu de la place, de part et d'autre du fleuve, il y a des arbres de Vie qui fructifient douze fois, une fois chaque mois : et leurs feuilles peuvent guérir les païens. (Apocal. 22, $2:$ :1637)

Ce que l'on peut lire dans le Coran, à la Sourate xxiv, où le pouvoir de Dieu est représenté par les bienfaits des arbres, n'est pas très différent : 
Dieu est la lumière des cieux et de la terre. Sa lumière est semblable à une niche où se trouve une lampe. La lampe est dans un verre pareil à un astre étincelant qui s'allume grâce à un arbre béni : un olivier qui n'est ni de l'orient ni de l'occident et dont l'huile brillerait sans qu'un feu la touche ou peut s'en faut. Lumière sur lumière.

Dieu dirige vers sa lumière qui il veut. Il propose aux hommes des paraboles. Dieu connaît parfaitement toute chose. (Le Coran, Sourate XXIV, 35 : 1116)

Le pouvoir surnaturel affirmé ici ne contredit en rien le poids attribué à la parole et à sa valeur de sublimation, comme le laisse entrevoir le Livre des Proverbes : "Langue apaisante est un arbre de vie, langue fourchue brise le cœur. » (Prov. 15, 4 : 820).

L'arbre démoniaque, qui a institué le péché et qui peut être considéré comme les «arbres de fin de saison, sans fruits, deux fois morts, déracinés » (Jud. 12: 1614), servira toujours de contrepoint indispensable pour traduire l'ambivalence essentielle du sujet et de ses corrélats, le pouvoir idéal de mort et de résurrection :

E de súbito sou tão feliz e tão forte que ouso inventar a história de uma criança morta. Alguém, não animal, não um espírito, alguém que não é ninguém porque é apenas uma boca, devora uma criança e corta-a em pedaços. Lóia pega nos pedaços e a criança cortada fica outra vez inteira, como a luz cortada ressuscita, como o galo morto cantou outra vez no quintal. (Gersão, $1997: 35-36)^{17}$.

3 La certitude de vaincre la mort, présente dans cet extrait, conjuguée avec l'indication tacite de la toute-puissance divine, qui prévoit l'existence d'un lieu utopique pour les justes, comme par exemple dans la Sourate LVI (« Ils seront parmi des lotus sans épines et des lits d'acacias ", Le Coran, Sourate LVI, 28-29:1760) et dans le livre d'Isaïe (" Au lieu d'épines croîtra le cyprès, au lieu d'orties croîtra le myrte », Is. 55, 13 : 1044), semble vouloir s'introduire dans le discours hypothétique de Gita, qui construit au mode conditionnel ce lieu sans épines mais avec les indispensables rangées d'arbres, éléments doublement constitutifs du décor :

Lóia estava longe mas eu aceitava perdê-la. Não se tinha dissolvido no ar, nem transformado em pássaro, areia ou folha. Existia, existia algures, Mocímboa da Praia era um lugar real, e isso era tranquilizador. Ficava ao norte, podia ver-se no mapa. Teria fileiras de coqueiros plantados. O vento bateria nas folhas. (Gersão, 1997 : 198-199) ${ }^{18}$

Le conditionnel, définissant un lieu inexistant mais possible, se transforme en une sorte d'indicateur de l'espace qui ne subsistera que dans la mémoire. Le départ de Gita pour Lisbonne, abandonnant les arbres, symboles de la nature et des personnes qui lui sont intimement liées, impliquera la réitération du mode conditionnel (qui ne sera jamais indicatif, futur ou présent) et l'acceptation d'un arbre rêvé et non perçu concrètement :

Um mundo que fica para trás (...) uma árvore que crescia nos sonhos e chegava ao céu - que sabem eles disso, que podem eles compreender ? (...) Vai faltar-me o ar em Lisboa, digo. (ibid. : 239) ${ }^{19}$

L'Afrique (et ses arbres) s'opposera à Lisbonne (supposée sans arbres), lieu inconnu et inhospitalier. L'arbre à paroles s'épuise avec le départ, la maturation de la jeune fille, dont l'initiation a commencé dans le jardin parmi les arbres qui dansaient (« As coisas, no quintal, dançavam ", ibid. : $9^{20}$ ), est complète et se termine par la disparition de son ami Roberto de son champ de vision, l'entrée dans la maison puis l'embarquement dans l'avion. C'est par la bouche de Gita que nous connaissons son milieu familial et le paysage qui l'entoure, que nous découvrons les différences de mode d'action et d'engagement avec l'Afrique, que nous comprenons comment l'arbre détient un pouvoir fondateur dans le déploiement de la parole, son simulacre (im)parfait. 
Bien que perdants (Amar Sanchez 2010), les personnages de A Árvore das Palavras parviennent à exorciser, grâce aux paroles de Gita et de ses avatars, un grand nombre des fantômes qui rendaient leur vie infernale, en créant tacitement mais sûrement un univers parallèle, pas toujours représentable, où plusieurs types d'arbres organisent progressivement le discours de façon à interférer avec le sens global.

\section{BIBLIOGRAPHIE}

AMAR SÁNCHEZ, Ana Maria (2010). Instrucciones para la Derrota - Narrativas éticas y Políticas de

Perdedores. Barcelona : Anthropos Editorial.

CRÉPON, Pierre (1991). Les Fleurs de Boudha - Anthologie du Boudhisme. Paris : Albin Michel.

ENCYCLOPÉDIE DES SYMBOLES (1996). Édition française sous la direction de Michel Cazenave. Paris : La Pochotèque, « Le Livre de Poche ».

GERSÃO, Teolinda (1997). A Árvore das Palavras. Lisboa : Publicações Dom Quixote.

LA SAINTE BIBLE (1961). Traduite en français sous la direction de L'École Biblique de Jérusalem. Paris : Les Éditions du Cerf.

LE CORAN (1979). Texte, traduction française et commentaire d'après la tradition, les différentes écoles de lecture, d'exégèse, de jurisprudence et de théologie, les interprétations mystiques, les tendances schismatiques et les doctrines hérétiques de l'Islam, et à la lumière des théories scientifiques, philosophiques et politiques modernes par Le Cheikh Si Hamza Boubakeur. Paris : Librairie Arthème Fayard, 2 vols.

ossola, Carlo (2011). En Pure Perte. Paris : Rivages Poche / Petite Bibliothèque.

TOMASSET, Claude \& PAGAN, Martine (dir.) (2011). Cacher, Se Cacher au Moyen Âge. Paris : Presses de l'Université Paris-Sorbonne.

\section{NOTES}

1. Notre traduction : « On restait longtemps sous l'arbre, adossé au tronc, et comme je l'ai dit, on se transformait en arbre. Ou bien en oiseau, bien que voler soit plus difficile. Mais c'était facile d'être les choses. Parce qu'on avait soudain en main la racine de tout ce qui était vivant. »

2. idem : "a planté lui-même un ricin au fond du jardin »

3. idem: «Il poussait en rêve, dis-je à Roberto alors que nous peignions l'écriteau. L'arbre à paroles. Pour contourner son tronc, il faudrait neuf lunes. Et chaque feuille était aussi grande qu'un vol d'oiseau. »

4. idem : «(...) la silhouette élancée des arbres, la Terre est une planète désolée et morte, en train de surnager.»; «j'irais jusqu'au bout de la ville de ciment et je continuerais de marcher parmi l'ombre rare, des arbres épars.»; «Foulant, sur le sol, les ombres menues, mouvantes, des cocotiers. » 
5. idem : «Ou bien je m'asseyais sous l'arbre du jardin et je parlais avec le vent et les feuilles. L'arbre agitait ses branches et je pensais : l'arbre à paroles. »

6. idem : « je me cachai derrière l'arbre le plus proche ».

7. idem : "Tronc en fleur tend ses branches à la jeunesse qui passe ».

8. idem : «Tu es bon comme les arbres sont des arbres et la pluie est la pluie ».

9. idem: «Je suis, disait l'arbre en agitant ses branches, la graine qui s'ouvre dans l'obscurité, l'eau qui se putréfie dans les marais, la forêt qui dort. Je suis ».

10. idem : «Le corps était l'arbre et le corps était le vent ».

11. idem : «La danse, c'est ça, une façon plus intense d'exister. Les arbres dansent, leurs feuilles dansent, la pluie danse, les animaux dansent, le soleil et la lune dansent. »

12. idem : « je continue à danser pieds nus, en frappant des mains, sous les arbres du jardin. »

13. idem : " on ne peut dompter ni les casuarines, ni les cocotiers, ni les jacarandas »

14. idem : «Je lève la tête vers les acacias de la rue où nous marchons, comme s'ils pouvaient nous donner une réponse, comme s'ils pouvaient nous indiquer un endroit mieux abrité que derrière un rideau de feuillage »; "Le vent souffle dehors, les arbres frissonnent de peur »; " Mais si elle pleurait, ce serait pire, sentit-elle en sortant un mouchoir en papier de son sac à main, c'était comme si le monde riait d'elle, comme si les parasols, les maisons, les bateaux, les arbres, les gens riaient d'elle. "

15. idem : «Un oiseau qui tombe de l'arbre parce qu'il ne s'est pas envolé à temps. »

16. idem : «Parfois, cet arbre [l'arbre à paroles] réapparaissait en rêve : il poussait au bord d'un cours d'eau et ses branches allaient jusqu'au ciel.», comme «le corps [qui] reliait la terre au ciel. »

17. idem : «Et soudain, je suis si heureuse et si forte que j'ose inventer l'histoire d'un enfant mort. Quelqu'un, pas un animal, pas un esprit, quelqu'un qui n'est personne parce que ce n'est qu'une bouche qui dévore un enfant et le coupe en morceaux. Lóia prend les morceaux et l'enfant découpé redevient entier, comme la lumière coupée ressuscite, comme le coq mort a de nouveau chanté dans le jardin. »

18. idem : «Loía était loin mais j'acceptais de la perdre. Elle ne s'était pas dissoute dans l'air, ni transformée en oiseau, en sable ou en feuille. Elle existait, elle existait quelque part, Mocimboa da Praia était un endroit réel, et c'était rassurant. Cela se trouvait au Nord, on pouvait le voir sur la carte. Il y aurait des rangées de cocotiers plantés. Le vent claquerait dans les feuilles. »

19. idem : «Un monde que l'on quitte (...) un arbre qui poussait en rêve et atteignait le ciel - que savent-ils de cela, que peuvent-il comprendre ? (...) Je vais manquer d'air à Lisbonne, dis-je. » 20. idem : «Les choses, dans le jardin, dansaient. »

\section{RÉSUMÉS}

Le roman de Teolinda Gersão, écrivain portugaise de la deuxième moitié du xxe siècle, joue avec les notions ambigües qui peuvent être associées au terme arbre. Sachant que l'arbre que l'on voit dans le paysage et que l'on peut étudier dans la botanique n'épuise pas toutes les significations inhérentes au concept, l'auteure se complaît à profiter de tous les sens cachés et de tous les symboles culturels qui peuvent être présents. Le roman, apparemment simple, devient de plus en plus complexe du moment où l'arbre assume une grande importance dans l'intrigue. Les personnages, en perte absolue, s'entremêlent au paysage et ne cessent d'identifier les arbres avec 
les avatars et les problèmes de leur existence. L'ancienne colonie portugaise, Mozambique, est la scène où se déroulent les événements et où se choquent les personnages plus ou moins adaptés à la vie africaine.

The novel by Teolinda Gersão, a Portuguese writer from the second half of the 20th century, plays with ambiguous notions that can be associated with the term tree. Knowing that the tree that we see in the landscape and that we study in botany does not exhaust all the meanings inherent in the concept, the author takes pleasure in taking advantage of all the hidden senses and all the cultural symbols that may be present. The seemingly simple novel becomes more and more complex as the tree assumes great importance in the plot. The characters, in absolute loss, intermingle with the landscape and continue to identify the trees with the avatars and the problems of their existence. The former Portuguese colony, Mozambique, is the scene where events take place and where characters more or less adapted to African life clash.

\section{INDEX}

Mots-clés : arbre, enfance, Afrique, colonie portugaise, monde féminin

Keywords : tree, childhood, Africa, Portuguese colony, female world

\section{AUTEUR}

\section{MARIA DE FÁTIMA MARINHO}

Universidade do Porto

fmarinho[at]letras.up.pt 